\title{
Dynamic Hedging with Stochastic Differential Utility*
}

\author{
Rodrigo De Losso da Silveira Bueno**
}

\begin{abstract}
In this paper we study the dynamic hedging problem using three different utility specifications: stochastic differential utility, terminal wealth utility, and a new utility transformation which includes features from the two previous approaches. In all three cases, we assume Markovian prices. While stochastic differential utility (SDU) has an ambiguous effect on the pure hedging demand, it does decrease the pure speculative demand, because risk aversion increases. We also show that in this case the consumption decision is, in some sense, independent of the hedging decision. In the case of terminal wealth utility (TWU), we derive a general and compact hedging formula which nests as special cases all of the models studied in Duffie and Jackson (1990). In the case of the new utility transformation, we find a compact formula for hedging which encompasses the terminal wealth utility framework as a special case; we then show that this specification does not affect the pure hedging demand. In addition, with CRRA- and CARA-type utilities the risk aversion increases, and consequently, the pure speculative demand decreases. If futures prices are martingales, then the transformation plays no role in determining the hedging allocation. Our results hold for a number of different price distributions. We also use semigroup techniques to derive the relevant Bellman equation for each case.
\end{abstract}

Keywords: Stochastic Control, Recursive Utility, Hedging, Bellman Equation.

JEL Codes: C61, D92, G11.

${ }^{*}$ Submitted in October 2005. Revised in September 2006. I thank the following persons for their comments: two anonymous referees, Lars Hansen, Olaf Korn, Jose Mazoy, Raghu Suryanarayanan, the seminar participants at USP, PUC-RJ, and EPGE, and the conference participants at the EFMA 2003 Annual Conference. I am responsible for any remaining errors.

${ }^{* *}$ Department of Finance, School of Management of São Paulo from the Getulio Vargas Foundation. Phone: 5511 3281-7859. E-mail: rdbueno@fgvsp.br

Brazilian Review of Econometrics

v. $26, \mathrm{n}^{\circ} 2$, pp. $257-289 \quad$ Nov 2006 


\section{Introduction}

Dynamic rather than static hedge improves hedging efficiency, meaning that it tends to increase utility. Empirically, it is difficult to believe a hedger would take a position and keep it until maturity. Indeed, as more information comes in, then the agent may be willing to rebalance his assets aiming at obtaining more protection (see Lien and Tse (2003), for further details and developments). In this paper we study the optimal futures hedging problem in a continuoustime setting with stochastic differential utility (Duffie and Epstein, 1992), SDU, assuming Markovian prices as in Adler and Detemple (1988). We extend the model in Duffie and Jackson (1990) - henceforth referred to as DJ - in three ways. First, we maximize the intertemporal consumption, in the spirit of Ho (1984). The consumption approach is interesting because in an intertemporal context the agent is willing to stabilize the expected consumption stream. For this purpose, the agent adjusts present consumption in order to allow investment intended for future consumption and also holds positions in futures. The latter is called futures hedging and the former consumption hedging.

Recursive utility (which is the class of utilities SDU belongs to) disentangles risk aversion from intertemporal substitutability, thereby permittting greater efficiency of the hedging strategy by means of its less restrictive structure. Our interest is to provide a new framework that may be potentially more efficient than without stochastic differential utility. In this sense, SDU adds some degrees of freedom ${ }^{1}$ in a possible empirical treatment still to be undertaken. Moreover, we would like to answer whether the futures position changes and in what direction in terms of buying more or less futures contracts. Consequently, we think of this approach as a tool which hedgers may use in order to improve the performance of their futures position. With standard ${ }^{2}$ SDU, however, the formula that we obtain depends on the derivatives of the value function, which are difficult to figure out. Therefore, in order to obtain the optimal hedge, we may have to employ numerical methods.

In our second extension of DJ the agent maximizes the terminal wealth; this is a usual assumption when our only concern is with a specific future date. Maximizing the utility of the terminal wealth has the advantage of producing closed formulas for some natural cases which can therefore be analyzed in greater depth. We are in fact able to produce a general hedging formula which nests as special cases all of the models studied in DJ. Then we specialize it to compare with those obtained by the authors.

As a consequence of regarding prices as Markov processes, the problem of myopic hedging at each time ${ }^{3}$ no longer necessarily occurs. Hence, our optimal

\footnotetext{
${ }^{1}$ In the econometric sense, it decreases the degrees of freedom, but here we are referring to the flexibility of the model to adjust for other possible specifications.

${ }^{2}$ As opposed to terminal wealth utility, which we discuss ahead.

${ }^{3}$ Myopic hedging at each time means that an agent is hedging only local changes in wealth. Further discussion can be found in Adler and Detemple (1988) and in the references therein.
} 
futures hedging formulas do not coincide with the corresponding static hedges and are not directly comparable with analogous solutions in discrete-time cases, as in the results in Anderson and Danthine (1981, 1983). Moreover, Markovian prices allow for a richer class of price distributions as opposed to DJ that had either martingales or independent normally distributed increments. If prices can be better modeled, then we may expect a superior hedging response when compared with DJ's model.

Our third extension of DJ is an attempt to connect the two approaches discussed before. We do this by making a suitable transformation on the Terminal Value-type utility. We then use the compact formulas that it produces to examine the impact of the transformation on the standard model. That is, inspired by the SDU model, we introduce the certainty equivalence machinery into the HamiltonJacobi-Bellman (HJB) equation of the terminal value utility, and then study the impact this has on the optimal hedge ratio.

The aforementioned connection between the SDU and the Terminal Utility is provided by the particular concave transformation we employ. This modification allows for additional degrees of freedom and may potentially improve the hedging strategy. Hence, the main advantage of this framework is that it encompasses the DJ model as a special case, makes it easy to implement and/or simulate, and allows us to derive some interesting, interpretable and neat results.

An important insight provided by the model is the following. To the best of our knowledge, real hedging positions have never been tested against the theory. All empirical works we are aware of estimate the hedging ratio and compare it with some benchmark, also artificially constructed. Now suppose you had real hedging position along some time and you estimated the determinants of this hedging position. Then by allowing SDU one has more elements to explain the results than otherwise.

Our model is similar to that of DJ in several aspects: spot and futures prices are vector diffusion processes; a hedge is a vector stochastic process which specifies a futures position in each futures market; and the hedging profits and losses are marked to market in an interest-paying margin account. The optimal hedge ratio is obtained by maximizing the expected utility, which is composed of a committed portfolio of spot markets together with the accumulated value of the margin account. However, we point out that - differently from what occurs in the DJ model - our results hold for a number of different price distributions.

The paper is organized as follows: in Section 2 we specify our model; in Section 3 we derive the optimal hedge under SDU; in Section 4 we derive the optimal hedge ratio maximizing only the terminal utility and examine special cases in order to compare our results with some of those obtained by DJ and also with our previous results; in Section 5 we derive the optimal hedge ratio under the proposed utility transformation and compare it with our previous results; finally, in Section 6 we conclude our discussion. In the Appendix we use semigroup techniques to obtain 
the relevant HJB equations; we state the proof of each proposition from the main text and explain some of the mathematical concepts that we use to derive the optimal hedge.

\section{The Model}

As mentioned earlier, our model (and this section as a whole) is very similar to DJ in that we consider an agent who chooses a future trading strategy so as to maximize the expected utility of consumption from $t$ to a future time $T \in \mathbb{R}_{+} \cup \infty$, according to the following setup

1. Let $B=\left(B^{1}, B^{2}, \ldots, B^{N}\right)^{\prime}$ denote a Standard Brownian Motion in $\mathbb{R}^{N}$ which is a martingale with respect to the agent's filtered probability space. ${ }^{4}$ We assume throughout the paper that all probabilistic statements are in the context of this filtered probability space.

2. $V$ denotes the space of predictable square-integrable processes ${ }^{5}$ such that

$$
V \equiv\left\{\text { predictable } v:[0, T] \times \Omega \rightarrow \mathbb{R} \mid E\left[\int_{0}^{t} v_{s}^{2} d s\right]<\infty, t \in[0, T]\right\}
$$

where $\Omega$ is the state space. Here predictable means measurable with respect to the $\sigma$-algebra generated by left-continuous processes adapted to the agent's filtration; that is, $v_{t}$ depends only on the information available up to time $t$.

3. There exist $M$ assets to be hedged, whose value is given by an $M$-dimensional Markov process $S$ with stochastic differential representation

$$
d S_{t}=\mu_{t}\left(S_{t}\right) d t+\sigma_{t}\left(S_{t}\right) d B_{t}
$$

where $\mu$ is $M$-dimensional, $\sigma$ is $(M \times N)$-dimensional and $\mu^{m} \in V$ and $\sigma^{m n} \in V$ for all $m$ and $n$ (thus, the Markov process $S$ is well defined). ${ }^{6}$

4. There are $K$ futures contracts available for trade at each instant of time, whose prices are given by a $K$-dimensional Itô process $F$ with stochastic differential representation

$$
d F_{t}=m_{t}\left(F_{t}\right) d t+v_{t}\left(F_{t}\right) d B_{t}
$$

\footnotetext{
$4 " \prime "$ indicates transpose, or differentiation when there is only one argument in the function. ${ }^{5}$ If $T=\infty$, then the square integrability condition is replaced by $E\left[\int_{0}^{\infty} e^{\beta t} v_{s}^{2} d s\right]<\infty$ where $\beta$ is the constant characterized in Appendix C of Duffie and Epstein (1992).

${ }^{6}$ Henceforth, we omit the dependence of the parameters on $S_{t}$ for simplicity.
} 
where $m^{k} \in V$ and $v^{k n} \in V$ for all $k$ and $n .^{7}$

5. A futures position is taken by marking to market a margin account according to a $K$-dimensional process $\theta=\left(\theta^{1}, \theta^{2}, \ldots, \theta^{K}\right)^{\prime}$, where $\theta^{\prime} m$ as well as each element of $\theta^{\prime} v$ belong to $V$. The space $\Theta$ of all such futures position strategies is then given by

$$
\Theta=\left\{\theta \mid \theta^{\prime} m \in V \text { and } \theta^{\prime} v^{n} \in V, \forall n\right\}
$$

6. At time $t$ the position $\theta_{t}$ in the $K$ contracts is credited with any gains or losses incurred by futures price changes, and the credits (or debits) are added to the agent's margin account. Thus, the margin account's current value, denoted by $X_{t}^{\theta}$, is credited with interest at the constant continuously compounding rate $r \geq 0$. Furthermore, we assume that any losses which bring the account to a negative level are covered by borrowing at the same interest rate, and we ignore transactions costs and other institutional features. The initial margin account $X_{0}^{\theta}=X_{0}$ is given. In a continuous-time model, the margin account then has the form

$$
X_{t}^{\theta}=\int_{0}^{t} e^{r(t-s)} \theta_{s}^{\prime} d F_{s}
$$

indicating that the 'increment' $\theta_{s}^{\prime} d F_{s}$ to the margin account at time $s$ is reinvested at the rate $r$, implying a corresponding increment of $e^{r(t-s)} \theta_{s}^{\prime} d F_{s}$ to the margin account by time $t$. Applying Itô's lemma, its corresponding stochastic differential equation is

$$
d X_{t}^{\theta}=\left(r X_{t}^{\theta}+\theta_{t}^{\prime} m_{t}\right) d t+\theta_{t}^{\prime} v_{t} d B_{t}
$$

7. Let $\pi_{t} \in \mathbb{R}^{M}$ be a bounded measurable function standing for the agent's spot commitment. Hence, the agent's total wealth at time $t$, given a futures position strategy $\theta$, is then $W_{t}^{\theta}$, where $W^{\theta}$ is the Itô process corresponding to the stochastic differential representation

$$
d W_{t}^{\theta}=\pi_{t}^{\prime} d S_{t}+d X_{t}^{\theta}-c_{t} d t
$$

where $c_{t} \in V$ is the consumption rate at time $t$.

8. The agent's consumption preferences at time $t$ are given by the stochastic differential utility ${ }^{8} U: V \rightarrow \mathbb{R}$, whose "aggregator", $(f, \kappa)$, is defined as

\footnotetext{
${ }^{7}$ Idem with respect to $F_{t}$.

${ }^{8}$ The next subsection provides more details on SDU.
} 
$f: \mathbb{R}^{V} \times \mathbb{R} \rightarrow \mathbb{R}$, and the variance multiplier, $\kappa$, as $\kappa: \mathbb{R} \rightarrow \mathbb{R}$. We assume $f$ to be regular, meaning that $f$ is continuous, Lipschitz in utility, and satisfies a growth condition in consumption. ${ }^{9}$ In addition, we assume that $f$ is increasing and concave in consumption. ${ }^{10}$ Consider the von NewmannMorgenstern index, $h$, which is continuous, strictly increasing, and satisfies a growth condition; then $h$ is in particular integrable (see Section 5 for examples). It is called the risk-adjustment function and measures the local risk aversion. Because we also adopt assumption $2^{11}$ of Duffie and Epstein (1992), we obtain $\kappa(J)=\frac{h^{\prime \prime}(J)}{h^{\prime}(J)}<0$. This yields the problem

$$
\max _{\theta \in \Theta, c \in V} E_{t} \int_{s \geq t}^{T}\left[f\left(c_{s}, J\left(z_{s}\right)\right)+\frac{1}{2} \kappa\left(J\left(z_{s}\right)\right) J_{z_{s}}^{\prime} \Sigma J_{z_{s}}\right] d s
$$

where $\delta>0$ is the subjective discount rate; ${ }^{12}$

$Z_{t}$ is a function that puts together both the processes $d W_{t}^{\theta}$ and $d X_{t}^{\theta}$, such that

$$
d Z_{t}=\mu_{z_{t}} d t+\Lambda_{z_{t}} d B_{t} \text { and }
$$

$\Sigma=\Lambda_{z_{t}} \Lambda_{z_{t}} \cdot{ }^{13}$

The function $f$ is generic, but it is simple to understand if you think of $\kappa(J)=0$ as described in footnote 12 . However, when $\kappa(J) \neq 0$, then things get very complex. Therefore, in the next subsection we provide more details regarding the role played by $\kappa(J)$ and how it is related to the value function. Duffie and Epstein (1992) show how the term $\frac{1}{2} \kappa\left(J\left(z_{s}\right)\right) J_{z_{s}}^{\prime} \Sigma J_{z_{s}}$ appears in the value function, whose origin comes from the application of Itô's lemma.

With this model in mind, we can define the optimal futures position.

\footnotetext{
${ }^{9}$ See DJ and Duffie and Epstein (1992:366) for more details on the meaning of these concepts. ${ }^{10}$ We can therefore freely apply Propositions 3 (monotonicity) and 5 (concavity) of DJ and Duffie and Epstein (1992).

${ }^{11}$ Assumption 2 in words means that the certainty equivalent measurable function at the mean of the stochastic variable is at least as great as at any other point. That is, the utility of an expected event is greater than the expected utility. This is the reason why $\kappa(J)<0$. Assumption 2 helps characterize the risk aversion of the stochastic differential utility (see Duffie and Epstein (1992:361) and 377).

12 Without recursive utility, $f\left(c_{s}, J\left(z_{s}\right)\right)=u\left(c_{s}\right)-\delta J\left(z_{s}\right)$, and $\kappa(J)=0$.

${ }^{13}$ Please, see further details in the proof of proposition 3.1.
} 
Definition 2.1 A futures position strategy $\theta$ is said to be optimal if it solves 2.6.

\subsection{Stochastic differencial utility: A prime}

The concept of stochastic differential utility is relatively new. Therefore this section aims at recovering the main features related to this kind of utility. However, we encourage the reader to refer to the original article. Here we follow Ait-Sahalia et al. (2004).

The stochastic differential utility formulation by Duffie and Epstein (1992) is a continuous-time analogue of the recursive utility model that appears in Epstein and Zin (1989). As it is well known, the main advantage of this framework relative to the standard one is that we can disentangle risk aversion from intertemporal substitutability. We now present the main characteristics of this approach, based on Duffie and Epstein (1992).

Define $J\left(z_{t}\right)$ to be the value function, where $z$ is the state variable. The standard additive utility specification of the utility at time $t$ for a consumption process $c$ is defined by

$$
J\left(z_{t}\right)=E_{t}\left[\int_{s \geq t} e^{-\delta(s-t)} u\left(c_{s}\right) d s\right], t \geq 0
$$

where $E_{t}$ denotes the expectation given the information available at time $t$, and $\delta$ is the discount rate.

The more general utility functions are called recursive; they exhibit intertemporal consistency and admit the Hamilton-Jacobi-Bellman characterization of optimality. The stochastic differential utility $U: V \rightarrow \mathbb{R}$ is defined as follows by two primitive functions $f: \mathbb{R}^{V} \times \mathbb{R} \rightarrow \mathbb{R}$ and $\kappa: \mathbb{R} \rightarrow \mathbb{R}$, as already explained. When well defined, the utility process $J$ for a given consumption process $c$ is the unique Itô process $J$ having a stochastic differential representation of the form

$$
d J\left(z_{t}\right)=\left[-f\left(c_{t}, J\left(z_{t}\right)\right)-\frac{1}{2} \kappa(J) J_{z_{t}}^{\prime} \Sigma J_{z_{t}}\right] d t+J_{z_{t}}^{\prime} \Lambda\left(z_{t}\right) d B_{t}
$$

where the subscript $J$ indicates derivative with respect to that argument.

In this framework the pair $(f, \kappa)$ is called the "aggregator"; it determines the consumption process $c$ such that the utility process $J$ is the unique solution to

$$
J\left(z_{t}\right)=E_{t}\left[\int_{s \geq t}\left\{f\left[c_{s}, J\left(z_{s}\right)\right]+\frac{1}{2} \kappa(J) J_{z_{s}}^{\prime} \Sigma J_{z_{s}}\right\} d s\right], t \geq 0
$$

We think of $J\left(z_{t}\right)$ as the continuation utility of $c$ at time $t$, conditional on current information; and of $\kappa(J)$ as the variance multiplier, applying a penalty (or reward) corresponding to a multiple of the utility "volatility" $J_{z}^{\prime} \Sigma J_{z}$. In a discretetime setting, we would say that at time $t$ the intertemporal utility $J(\cdot, t+1)$ for the period ahead and beyond is a random variable. Thus the agent first computes 
the certainty equivalence $\eta\left(\sim J(\cdot, t+1) \mid \Im_{t}\right)$ of the conditional distribution $\sim$ $J(\cdot, t+1) \mid \Im_{t}$ of $J(\cdot, t+1)$, given information $\Im_{t}$ at time $t$. Then (s)he combines the latter with $c_{t}$ via the aggregator. The function $f$ encodes the intertemporal substitutability of consumption and other aspects of "certainty preferences"; it also generates a collateral risk attitude under uncertainty. The certainty equivalence function, $\eta$, encodes the risk aversion in the sense described in Epstein and Zin (1989).

In this paper, our expected utility-based specification is given by

$$
\eta(\sim J)=h^{-1}\left(E_{t}[h(J)]\right)
$$

where $J$ is a real-valued integrable random variable, $\sim J$ denotes its distribution, and $h$ has been already defined in the last section.

\section{Stochastic Differential Utility}

In this section we directly derive the optimal futures hedging under Markovian prices and stochastic differential utility.

Proposition 3.1 The optimal futures position strategy is $\theta^{S D U}$, where

$$
\begin{aligned}
\theta_{t}^{S D U}= & -\frac{\left(J_{w w}+J_{w x}\right)+\kappa(J)\left(J_{w}^{2}+J_{w} J_{x}\right)}{\left(J_{w w}+2 J_{w x}+J_{x x}\right)+\kappa(J)\left(J_{w}^{2}+2 J_{w} J_{x}+J_{x}^{2}\right)} \times \\
& \left(v_{t} v_{t}^{\prime}\right)^{-1}\left[v_{t} \sigma_{t}^{\prime} \pi_{t}+\frac{J_{w}+J_{x}}{\left(J_{w w}+J_{w x}\right)+\kappa(J)\left(J_{w}^{2}+J_{w} J_{x}\right)} m_{t}\right]
\end{aligned}
$$

\section{Proof See Appendix B1}

The optimal strategy is characterized in terms of the derivatives of the value function as in Breeden (1984), Ho (1984), and Adler and Detemple (1988); we may therefore have to solve this equation using numerical methods. Notice that consumption does not explain the hedging decisions. In fact, by looking at Appendix $\mathrm{B} 1$, one can see that the optimal consumption is determined independently of the hedging decisions, a result that is similar to that of Ho (1984). A possible explanation for this independence is the possibility of borrowing or lending in order to adjust the margin account, leaving the consumption adjustments free.

Observe that the drift of the spot prices does not explicitly affect the optimal futures hedging. It may have an indirect effect, however, through the derivatives of the Bellman equation, for instance. The utility parameters, the volatility parameters of the prices, and the drift of the futures prices affect the optimal strategy in an obvious way. Of course, it is easy to see that if $\kappa(J)=0$ then we obtain the optimal futures hedging formula for the standard additive utility specification. ${ }^{14}$

\footnotetext{
${ }^{14}$ This occurs because of the independence of the consumption and hedging decisions. See also the hedging formula in Section 4.
} 
Under our assumptions on the utility and on the aggregator, the first coefficient is positive at the optimal point ${ }^{15}$

$$
A_{t} \equiv \frac{(\overbrace{J_{w w}+J_{w x}}^{-})+\overbrace{\kappa(J)}^{-}(\overbrace{J_{w}^{2}+J_{w} J_{x}}^{+})}{(\underbrace{J_{w w}+2 J_{w x}+J_{x x}}_{-})+\kappa(J)(\underbrace{J_{w}^{2}+2 J_{w} J_{x}+J_{x}^{2}}_{+})}>0
$$

Because we do not know the functional form of the value function, it is difficult to determine whether $A$ is greater or less than it would be under standard utility. We do know, however, that its sign does not change under SDU.

In addition, the term that multiplies $m_{t}$ is negative

$$
-R_{t}^{-1} \equiv \frac{\overbrace{J_{w}+J_{x}}^{+}}{(\underbrace{J_{w w}+J_{w x}}_{-})+\underbrace{\kappa(J)}_{-}(\underbrace{J_{w}^{2}+J_{w} J_{x}}_{+})}<0
$$

We interpret $R$ as an extended version of the risk aversion coefficient because it includes terms like $J_{x}$ and $J_{w x}$. The numerator is a measure of the global concavity of the sum of the value function with the term generated by the SDU approach; the denominator stands for the global curvature of the value function. ${ }^{16}$ If the value function becomes more concave, either due to the wealth or to the margin account, then $R$ increases as in the standard case. Furthermore, notice that the SDU utility adds a penalty which corresponds to the additional term in the numerator if the curvature of the value function increases, then both the numerator and the denominator increase, so the net effect is ambiguous (in the standard case, the risk aversion would decrease). The sign of $R$ does not change under SDU. Hence, the global net effect is an increase in the extended risk aversion due to the presence of the aforementioned term in the denominator.

With this in mind, Duffie (1989) calls the first term between brackets in equation 3.1 the pure hedge demand, and the second term the pure speculative demand. ${ }^{17}$ The term pure hedge demand comes from a single-period model in

\footnotetext{
${ }^{15}$ The first-order conditions imply $0<J_{w}=u^{\prime}(c)$, and, because $c$ is a normal good, we also have $\frac{\partial c}{\partial w}, \frac{\partial c}{\partial x}>0$

${ }^{16}$ With Terminal Utility, as we will see later, this term becomes the standard coefficient of risk aversion $-\frac{J_{w w}}{J_{w}}$.

${ }^{17}$ Adler and Detemple (1988) call them, respectively, the Merton/Breeden informationally based hedging component and the mean-variance component.
} 
which the only concern is with minimizing risk, that is, with minimizing the variance of the position without taking the return into account. In this case, $\theta_{t}=\left(v_{t} v_{t}^{\prime}\right)^{-1} v_{t} \sigma_{t}^{\prime} \pi_{t}$ (see Subsection 4.1 for further discussion).

Even if the spot commitment is zero at time $t$, the hedger may still be willing to buy futures due to the pure speculative demand term. This might also happen if the covariance between spot and futures prices is null $-v_{t}$ is orthogonal to $\sigma_{t}-$, meaning that futures contracts do not provide any protection against spot price fluctuations. ${ }^{18}$ Also, if the covariance between futures and spot prices increases in absolute value then one might want to increase the position under hedge, since in this case it becomes more urgent to protect oneself against undesirable fluctuations in prices.

Looking at the second term inside the brackets, the formula shows that the coefficient of $m_{t}$ increases with the extra term in the denominator, since as we have shown the entire expression is negative. Thus, its absolute value decreases. Therefore the contribution of the pure speculative demand to the optimal hedge strategy decreases with recursive utility. If $m_{t}=0$, however, the solution does not depend explicitly on the drift of futures prices, but instead - and unlike what occurs in DJ - it depends on the parameters of the recursive utility via $\kappa(J)$ and the other derivatives.

\section{Terminal Wealth}

The model we consider in this section is very similar to the model that was discussed before. We do, however, introduce a fundamental modification: we maximize the utility of the terminal wealth instead of consumption over time. We consequently consider here a finite time $T$. We also make the simplifying assumption of taking the spot commitment constant over time. This makes our model identical to that of DJ, except that our prices are Markovian. This assumption about the prices is not new; Adler and Detemple (1988) have used it in a model constructed to solve a similar problem. Formally, we have

1. The agent is committed to receiving the value at time $T$ of a position in the assets represented by a fixed portfolio $\pi \in \mathbb{R}^{M}$, yielding the terminal value $\pi^{\prime} S_{T}$. Hence, the total wealth of the agent at time $T$, given a futures position strategy $\theta$, is $W_{T}^{\theta}$, where $W^{\theta}$ is the Itô process with stochastic differential representation

$$
d W_{t}^{\theta}=\pi^{\prime} d S_{t}+d X_{t}^{\theta}
$$

2. The preferences of the agent over wealth at time $T$ are given by a von Newman-Morgenstern utility function $U: \mathbb{R} \rightarrow \mathbb{R}$, which is monotonic, twice

\footnotetext{
${ }^{18}$ Here we are not taking equilibrium concerns into account.
} 
continuously differentiable, and strictly concave, with $U^{\prime}$ and $U^{\prime \prime}$ each satisfying a (linear) growth condition. This yields the problem

$$
\max _{\theta \in \Theta} E_{t}\left[U\left(W_{T}^{\theta}\right)\right]
$$

Obviously, the optimal position in this case is the one that maximizes the expression 4.9. We can now state the proposition

Proposition 4.1 The optimal futures position strategy for maximizing the terminal utility is $\theta^{T V}$, where

$$
\theta_{t}^{T V}=-\frac{\left(J_{w w}+J_{w x}\right)}{\left(J_{w w}+2 J_{w x}+J_{x x}\right)}\left(v_{t} v_{t}^{\prime}\right)^{-1}\left[v_{t} \sigma_{t}^{\prime} \pi_{t}+\frac{J_{w}+J_{x}}{\left(J_{w w}+J_{w x}\right)} m_{t}\right]
$$

\section{Proof See Appendix B2}

Notice that we are only concerned with the local mean effect, because we are interested in the terminal value of the wealth at the maturity of the contract. Also, observe the similarity of this expression with that obtained under SDU, had we assumed $\kappa(J)=0$.

The qualitative analysis we provided before regarding the formula under SDU holds here without modification, so we shall not repeat it.

Surprisingly, this formula can be compacted further. In order to do so, let us state an important property of the model

Proposition 4.2 Given a variation in the initial wealth, the net return of the variation in the final wealth is equal to the variation in the final wealth given a variation in the initial margin account. Formally,

$$
\{\exp [r(T-t)]-1\} \frac{\partial W_{T-t}^{\theta_{t}}}{\partial w}=\frac{\partial W_{T-t}^{\theta_{t}}}{\partial x}
$$

\section{Proof See Appendix B3}

Proposition 4.2 tells us that a positive variation in the initial wealth implies a positive variation in the final wealth, and hence in the value function, as we shall soon see. A similar argument can be made with respect to the initial position in the margin account. Notice in the proof that an increase in the initial margin account amounts to a gross return growth in the final margin during the period considered.

This result does not rely on the utility assumptions, but only on the budget constraint. Consequently, it holds whether we are at the optimal point or not. 
The next statement is a direct consequence of this proposition. It shows a connection between the derivatives of the value function.

Corollary 4.1 Given the assumptions on the utility and budget constraint, then the following equality holds

$$
\{\exp [r(T-t)]-1\} J_{w}=J_{x}
$$

Proof See Appendix B4

From Corollary 4.1 we obtain the following interesting consequence:

Corollary 4.2 The following equality holds

$$
\{\exp [r(T-t)]-1\}^{2} J_{w w}=\{\exp [r(T-t)]-1\} J_{w x}=J_{x x}
$$

Proof See Appendix B5

Observe that all second derivatives are negative, since $U\left(W_{T-t}^{\theta_{t}}\right)$ is assumed concave.

Now, by substituting in the relationships that we obtained in the optimal hedging equation we find the following, more compact, hedge ratio.

Proposition 4.3 Given our assumption on the budget constraint and utility, the optimal hedging ratio is given by

$$
\theta_{t}^{T V}=-\exp [-r(T-t)]\left(v_{t} v_{t}^{\prime}\right)^{-1}\left[v_{t} \sigma_{t}^{\prime} \pi+\frac{J_{w}}{J_{w w}} m_{t}\right]
$$

Proof Replace terms.

The main novelty here is that the first - and rather large - term outside the brackets reduces to a deterministic expression which does not depend on the form of the value function.

In order to further sharpen our analysis, in the next subsections we make some simplifying assumptions and then show how our results are related to those of DJ.

\subsection{Martingale futures prices $-\mathrm{m}_{\mathrm{t}}=\mathbf{0}$}

Our first special case occurs when the drift of the futures price is identically equal to zero. This also happens in four out of the five cases studied in DJ.

Proposition 4.4 Under our assumptions, together with $m_{t}=0$, the optimal futures position strategy is given by $\theta^{T V}$, where

$$
\theta_{t}^{T V}=-e^{-r(T-t)}\left(v_{t} v_{t}^{\prime}\right)^{-1} v_{t} \sigma_{t}^{\prime} \pi
$$


This result is very similar to cases 1, 3 and 4 in DJ, all of which assume martingale futures prices. In case 1, they also assume Gaussian prices; in case 3, mean-variance preferences; and in case 4 log-normal spot prices and mean-variance utility. Here we obtain their results under more general assumptions. The bad news is that, if $m_{t}=0$, then the utility parameters play no role in the formula. The explanation given by DJ is that the demand for futures is based only on the hedge they provide, such that futures are only used to control 'noise' in the portfolio process. In the words of DJ, "the optimal manner of doing so depends solely on the structure of the 'noise' in the price process, not on the structure of the utility, nor on the drift of the assets' price processes".

In particular, let us see what happens if we specify martingale futures prices and log-normally distributed spot prices. This is a very special case, and in order to make it tractable let us assume the following condition for the $m$-th spot price

$$
d S_{t}^{m}=g_{t}^{m} S_{t}^{m} d t+S_{t}^{m} h_{t}^{m \prime} d B_{t}
$$

In addition, in order to make this process log-normally distributed we assume $g_{t}^{m}$ and $h_{t}^{m}$ to be deterministic, where $g_{t}^{m}:[0, T] \rightarrow \mathbb{R}$ and $h_{t}^{m}:[0, T] \rightarrow \mathbb{R}^{N}$ are bounded measurable functions.

Proposition 4.5 Under martingale futures prices and log-normally distributed spot prices, the optimal hedge ratio is

$$
\theta_{t}^{T V}=-e^{-r(T-t)}\left(v_{t} v_{t}^{\prime}\right)^{-1} v_{t} H_{t}^{\prime} \pi
$$

where $H_{t}$ is the $M \times N$ matrix whose $m^{\text {th }}$ row is $S_{t}^{m} \exp \left[\int_{t}^{T}\left(g_{s}^{m}-\frac{1}{2} h_{s}^{m \prime} h_{s}^{m}\right) d s\right]$ $h_{t}^{m \prime}$.

\section{Proof See DJ.}

\subsection{Exponential utility $-\mathrm{m}_{\mathrm{t}} \neq \mathbf{0}$}

Exponential utility consists in assuming $u(w)=-e^{-\gamma w}$, where $\gamma>0$ is a constant measure of risk aversion. This type of utility is often used in continuoustime investment studies.

Proposition 4.6 Under exponential utility, the optimal futures position strategy is given by $\theta^{*}$, where

$$
\theta_{t}^{*}=-e^{-r(T-t)}\left(v_{t} v_{t}^{\prime}\right)^{-1}\left[v_{t} \sigma_{t}^{\prime} \pi-\frac{1}{\gamma} m_{t}\right]
$$

Proof See Appendix B6 
This coincides with case 2 of DJ, as expected. Here it is easy to see that if the risk aversion increases then the pure speculative demand on the optimal hedge decreases.

We could consider other commonly used utility functions, such as power and quadratic functions. But we would then have to deal with two problems. First, power functions, for example, do not admit negative values for wealth. A second and more practical problem is that in such cases we would have to use numerical methods to find the optimal hedge, because the expected wealth that would appear in the RHS depends on the optimal hedging strategy.

We conclude this subsection by remarking that one could combine the assumptions that we have derived individually in order to obtain a more comprehensive optimal hedging strategy.

\section{Connecting SDU and Terminal Utility}

We would like to make a connection between the two approaches that we have studied. We may do this by making a suitable transformation on the Terminal Value-type utility. Once we do so, we can then use the compact formulas generated by the second-type utility in order to explicitly determine the effects of the SDU model on the optimal hedge ratio. ${ }^{19}$ The SDU framework motivates us to add the certainty equivalence machinery to the Terminal Wealth framework. A practical reason for us to do so is to increase the flexibility of the model, and hence to increase the efficiency of the hedge. In order to achieve this we consider a particular concave transformation of the terminal value-type utility. Formally we then have

1. The preferences of the agent over wealth at time $t$ are given by the utility $U: V \rightarrow \mathbb{R}$ defined by ${ }^{20}$

$$
\max _{\theta \in \Theta} h^{-1}\left(E_{t}\left[h\left(U\left(W_{T}^{\theta_{t}}\right)\right)\right]\right)
$$

The practical advantage here is that we obtain neat formulas for our problem. From the theoretical point of view we are assuming that we can add the certainty equivalence in the HJB equation when we are maximizing the utility of the final wealth. Our procedure relies on the remarkable consequence of the forwardlooking nature of the Bellman equation, which makes state variables reflecting past decisions unnecessary (Duffie and Epstein (1992:373)).

We can now obtain the optimal hedging ratio

\footnotetext{
${ }^{19}$ Since there is no consumption stream, it does not make sense to speak of intertemporal substitution.

${ }^{20} \mathrm{An}$ alternative, and equivalent, characterization would be to maximize $J\left(z_{t}\right)=$ $E_{t}\left[U\left(W_{T}^{\theta t}\right)\right]+\frac{1}{2} E_{t} \int_{s>t}^{T} \kappa\left(J\left(z_{s}\right)\right) J_{z_{s}}^{\prime} \Sigma J_{z_{s}} d s$ (see the Appendix). This would generate the same HJB equation, and hence the same evolution of the value function. Any attempts at further analysis, however, would become much messier. This is the reason why we have chosen the definition in the main text.
} 
Proposition 5.1 The optimal futures position strategy is given by $\theta^{T W S D U}$, where

$$
\begin{aligned}
\theta_{t}^{T W S D U}= & -\frac{\left(J_{w w}+J_{w x}\right)+\kappa(J)\left(J_{w}^{2}+J_{w} J_{x}\right)}{\left(J_{w w}+2 J_{w x}+J_{x x}\right)+\kappa(J)\left(J_{w}^{2}+2 J_{w} J_{x}+J_{x}^{2}\right)} \times \\
& \left(v_{t} v_{t}^{\prime}\right)^{-1}\left[v_{t} \sigma_{t}^{\prime} \pi_{t}+\frac{J_{w}+J_{x}}{\left(J_{w w}+J_{w x}\right)+\kappa(J)\left(J_{w}^{2}+J_{w} J_{x}\right)} m_{t}\right]
\end{aligned}
$$

\section{Proof See Appendix Br}

This expression is similar to the formula found in Section 3. In addition, because this is a concave transformation, the lemmas proved in Section 4 also hold here. Let us point out the following

Lemma 5.1 Given the assumptions on utility and wealth in this section, then

$$
\begin{aligned}
0 & >\frac{J_{w}}{J_{w w}+\kappa(J) J_{w}^{2}}= \\
& =\frac{E_{t}\left[h^{\prime}\left(U\left(W_{T-t}^{\theta_{t}}\right)\right) U^{\prime}\left(W_{T-t}^{\theta_{t}}\right)\right]}{E_{t}\left[h^{\prime \prime}\left(U\left(W_{T-t}^{\theta_{t}}\right)\right)\left[U^{\prime}\left(W_{T-t}^{\theta_{t}}\right)\right]^{2}+h^{\prime}\left(U\left(W_{T-t}^{\theta_{t}}\right)\right) U^{\prime \prime}\left(W_{T-t}^{\theta_{t}}\right)\right]}
\end{aligned}
$$

Proof See Appendix B8

Consequently, the expression of the hedge ratio becomes much simpler.

Proposition 5.2 Given our assumptions on the budget constraint and on the utility, the optimal hedging ratio is given by

$$
\theta_{t}^{T W S D U}=-\exp [-r(T-t)]\left(v_{t} v_{t}^{\prime}\right)^{-1}\left[v_{t} \sigma_{t}^{\prime} \pi+\frac{J_{w}}{J_{w w}+\kappa(J) J_{w}^{2}} m_{t}\right]
$$

Proof Apply the corollaries of Section 4.

This formula shows that the certainty equivalence machinery does not affect the pure hedging demand, just as occurred in the case of terminal wealth. Of course, if $\kappa(J)=0$ then we return to the standard case. Note that $J_{w w}+\kappa(J) J_{w}^{2}<0$ as expected. But since we do not know the sign of $J_{w w}$, and since this is not an SDU setting, it is not obvious that the risk aversion increases. Moreover, if $m_{t}=0$ then the certainty equivalence structure has no effect on the optimal hedge ratio, and the conclusion in DJ that the demand for futures depends only on the hedge holds. 


\subsection{Exponential risk-adjustment CRRA and CARA}

In this section we provide two simple examples where we specify $h(u)=-e^{-\rho u}$, with $\rho>0$. In both examples we show that the risk aversion increases, causing the pure speculative demand to decrease.

Let us first assume that $u(w)=-e^{-\gamma w}$, where $\gamma>0$. Then ${ }^{21}$

$$
\begin{aligned}
R & =-\left(\frac{J_{w w}}{J_{w}}+\kappa(J) J_{w}\right)= \\
& =\gamma\left(1+\rho \frac{E_{t}\left[\exp \left(-\rho U-2 \gamma W_{T-t}^{\theta_{t}}\right)\right]}{E_{t}\left[\exp \left(-\rho U-\gamma W_{T-t}^{\theta_{t}}\right)\right]}\right)
\end{aligned}
$$

We may interpret $R$ as the global risk aversion parameter, just as we did in Section 3. By inspecting this expression one clearly sees that once certainty equivalence is introduced then numerical methods become necessary to find the optimal hedge ratio. One also sees that the risk aversion parameter increases by the factor $\rho \frac{E_{t}\left[\exp \left(-\rho U-2 \gamma W_{T-t}^{\theta_{t}}\right)\right]}{E_{t}\left[\exp \left(-\rho U-\gamma W_{T-t}^{\theta_{t}}\right)\right]}>0$. As a consequence, the pure speculative demand decreases. ${ }^{22}$ Moreover, if $\rho=0$, then we are back to the Terminal Value-type utility example discussed in the last section.

For our second example, suppose $u(w)=\frac{w^{1-\gamma}}{1-\gamma}, \gamma>0$. Then

$$
\begin{aligned}
R & =-\left(\frac{J_{w w}}{J_{w}}+\kappa(J) J_{w}\right)= \\
& =\gamma \frac{E_{t}\left[e^{-\rho U}\left(W_{T-t}^{\theta_{t}}\right)^{-1-\gamma}\right]}{E_{t}\left[e^{-\rho U}\left(W_{T-t}^{\theta_{t}}\right)^{-\gamma}\right]}\left(1+\frac{\rho}{\gamma} \frac{E_{t}\left[e^{-\rho U}\left(W_{T-t}^{\theta_{t}}\right)^{-2 \gamma}\right]}{E_{t}\left[e^{-\rho U}\left(W_{T-t}^{\theta_{t}}\right)^{-1-\gamma}\right]}\right)
\end{aligned}
$$

\footnotetext{
${ }^{21}$ We suppress the TWSDU superscript of $\theta$ for simplicity.

${ }^{22}$ We are not considering equilibrium effects.
} 
The risk aversion again increases, because $\frac{E_{t}\left[e^{-\rho U}\left(W_{T-t}^{\theta_{t}}\right)^{-1-\gamma}\right]}{E_{t}\left[e^{-\rho U}\left(W_{T-t}^{\theta_{t}}\right)^{-\gamma}\right]}>$ $\frac{E_{t}\left[\left(W_{T-t}^{\theta_{t}}\right)^{-1-\gamma}\right]}{E_{t}\left[\left(W_{T-t}^{\theta_{t}}\right)^{-\gamma}\right]}$ (see Appendix E for a proof), and also because of the additional term between parentheses. In particular, with log-utility, $\gamma=1$, the risk aversion coefficient increases by a factor somewhat greater than $\rho$.

\section{Conclusions}

In this paper we study the dynamic hedging problem using three different utility specifications: stochastic differential utility, terminal wealth utility, and a new utility which connects the two previous approaches through the common certainty equivalence structure. We assume Markovian prices, as in Adler and Detemple (1988), in all three cases. As a consequence of this assumption we avoid the myopic hedging problem at each time. Furthermore, depending on the specification of the utility function, we must use different Hamilton-Jacobi-Bellman (HJB) equations. Finally, this approach produces hedging formulas that - differently from what occurs in the Duffie and Jackson (1990) model - are valid for a number of different price distributions.

The stochastic differential utility (SDU) hypothesis, where the agent maximizes consumption over time as in Ho (1984), affects the pure hedging demand ambiguously, because SDU parameters increase both the denominator and the numerator of the optimal ratio. Nevertheless, SDU does decrease the pure speculative demand, because risk aversion increases. We also show that the consumption decision is independent of the hedging decision in the sense that we can split the program into two independent programs, one for the consumption and the other one for the optimal hedging. In the latter case, if the drift of futures prices is zero, then there is no clear impact on the optimal hedge.

In the case of the second type of utility, we derive a general and compact hedging formula which nests all cases studied in Duffie and Jackson (1990). This formula includes the following special assumptions found in their paper: Gaussian prices, mean-variance utility, and log-normal prices.

In our third case, that of the modified Terminal Utility, we find a compact formula for hedging which encompasses the second type of utility as a special case. We then show that the pure hedging demand is not affected by this specification. We also see that with CRRA- and CARA-type utilities the risk aversion increases and consequently the pure speculative demand decreases. If futures prices are martingales, then this modification plays no role in determining the hedging allocation, so we obtain the same strategy as in the Terminal Value model.

We believe that it would be very interesting to study the general equilibrium problem of this economy. That might be done theoretically and by means of simulations where one could study whether the equilibrium exists and if it is unique or multiple. 
In the Appendix, we use semigroup techniques in order to obtain the relevant Bellman equation for each case.

Although this is an essentially theoretical work, there are some possible empirical applications of our model. For instance, one might try to simulate the hedge ratio and compare the result with some benchmark, by varying the parameters of our model, provided some stationary assumptions are satisfied. Another possibility is to compare our model with alternative ones and try to infer its efficiency. In either case, we could study the results of having a hedger following the optimal hedging strategy suggested by our model. Indeed, we believe that hedgers might want to test the efficiency of this structure.

As in Duffie and Jackson (1990), we did not try to assess the implementation efforts of our model. However, given the present stage of computing technology, as well as the substantial gains in hedging efficiency that might be achieved, it might be worthwhile to make an effort in this direction in the future.

\section{References}

Adler, M. \& Detemple, J. B. (1988). On the optimal hedge of a non-traded cash position. The Journal of Finance, 43(1):143-153.

Ait-Sahalia, Y., L., H., \& Scheinkman, J. A. (2004). Operator methods for continuous-time Markov models. In Ait-Sahalia, Y., editor, Handbook of Financial Econometrics. Elsevier.

Anderson, R. W. \& Danthine, J.-P. (1981). Cross-hedging. Journal of Political Economy, 89:1182-1196.

Anderson, R. W. \& Danthine, J.-P. (1983). The time pattern of hedging and the volatility of futures prices. Review of Economic Studies, 50:249-266.

Breeden, D. (1984). Futures markets and commodity options hedging and optimality in incomplete markets. Journal of Economic Theory, 32:275-300.

Duffie, D. (1989). Futures Markets. Englewood Cliffs Prentice Hall.

Duffie, D. \& Epstein, L. G. (1992). Stochastic differential utility. Econometrica, 60(2):353-394.

Duffie, D. \& Jackson, M. O. (1990). Optimal hedging and equilibrium in a dynamic futures market. Journal of Economics Dynamics Control, 14:21-33.

Epstein, L. G. \& Zin, S. E. (1989). Substitution, risk aversion and the temporal behavior of consumption and asset returns. A theoretical framework. Econometrica, 57(4):937-969.

Ethier, S. N. \& Kurtz, T. G. (1986). Markov Processes Characterization and Convergence. Wiley, New York. 
Hansen, L. P. \& Scheinkman, J. A. (2002). Semigroup pricing. Manuscript, University of Chicago.

Ho, T. S. Y. (1984). Intertemporal commodity futures hedging and production decision. The Journal of Finance, 39(2):351-376.

Krylov, N. V. (1980). Controlled Difusion Processes. Apringer-Verlag, New York.

Lien, D. \& Tse, Y. K. (2003). Some recent developments in futures hedging. Journal of Economic Surveys, 16(3):357-396.

Oksendal, B. (2003). Stochastic Differential Equations. Springer-Verlag, New York, 6th. edition. 


\section{Appendix A - Infinitesimal Generators}

Here we discuss some technical concepts that we have used throughout the paper. We first present the infinitesimal generator approach, following closely AitSahalia et al. (2004). The HJB equation is easier to derive using this approach.

Let $(\Omega, \Im, P)$ denote a probability space, $\Xi$ a locally compact metric space with a countable basis $E$, a $\sigma$-field of Boreleans in $\Xi$, and $I$ an interval of the real line. For each $t \in I$, let $X_{t}$ be a stochastic process such that $X_{t}:(\Omega, \Im, P) \rightarrow(\Xi, E)$ is a measurable function, where $(\Xi, E)$ is the state space.

Definition A.1 $Q:(\Xi, E) \rightarrow[0, \infty]$ is a transition probability if $Q(x, \cdot)$ is a probability measure in $\Xi$, and $Q(\cdot, B)$ is measurable, for each $(x, B) \in(\Xi \times E)$.

Definition A.2 A transition function is a family $Q_{s, t},(s, t) \in I^{2}, s<t$ that satisfies the Chapman-Kolmogorov equation for each $s<t<u$

$$
Q_{s, u}(x, B)=\int Q_{t, u}(y, B) Q_{s, t}(x, d y)
$$

A transition function is homogeneous if $Q_{s, t}=Q_{s^{\prime}, t^{\prime}}$ whenever $t-s=t^{\prime}-s^{\prime}$.

Definition A.3 Let $\Im_{t} \in \Im$ be an increasing family of $\sigma$-algebras, and $X$ be a stochastic process that is adapted to $\Im_{t}$. $X$ is Markov with transition function $Q_{s, t}$ if for each non-negative Borel measurable $\phi: \Xi \rightarrow \mathbb{R}$ and each $(s, t) \in I^{2}, s<t$ we have:

$$
E\left[\phi\left(X_{t}\right) \mid \Im_{s}\right]=\int \phi(y) Q_{s, t}\left(X_{s}, d y\right)
$$

Assume that $Q_{t}$ is a homogeneous transition function and that $L$ is a vector space of real-valued functions such that for each test function $\phi \in L, \int \phi$ (y) $Q_{t}(x, d y) \in L$. Now, for each $t$ define the conditional expectation operator

$$
\mathcal{T}_{t} \phi(x)=E\left[\phi\left(y_{t}\right) \mid x_{0}=x\right]=\int \phi(y) Q_{t}(x, d y)
$$

The Chapman-Kolmogorov equation guarantees that the linear operators $\mathcal{T}_{t}$ satisfy $\mathcal{T}_{t+s}=\mathcal{T}_{t} \mathcal{T}_{t}$.

With this in hand we can propose a parameterization for the Markov processes. In order to do this, let $(L,\|\cdot\|)^{23}$ be a Banach space.

${ }^{23}$ Notice that $V$ is contained in this space. 
Definition A.4 A one-parameter family of linear operators in $L,\left\{\mathcal{T}_{t}: t \geq 0\right\}$ is a strongly continuous contraction semigroup if

a. $\mathcal{T}_{0}=I$

b. $\mathcal{T}_{t+s}=\mathcal{T}_{t} \mathcal{T}_{s}$ for all $s, t \geq 0$;

c. $\lim _{t \rightarrow 0} \mathcal{T}_{t} \phi=\phi ;$ and

d. $\left\|\mathcal{T}_{t}\right\| \leq 1$.

In general, the semigroup of conditional expectations determines the finitedimensional distributions of the Markov process, as we can infer from Ethier and Kurtz (1986), Proposition 1.6 of Chapter 4).

We can now define infinitesimal generators. A generator describes the instantaneous evolution of a semigroup. Heuristically, we could think of it as the derivative of the operator $\mathcal{T}$ with respect to time, when it goes to zero.

Definition A.5 The infinitesimal generator of a semigroup $\mathcal{T}_{t}$ on a Banach space $L$ is the (possibly unbounded) linear operator $\mathcal{A}$ defined by

$$
\mathcal{A} \phi=\lim _{t \downarrow 0} \frac{\mathcal{T}_{t} \phi-\phi}{t}
$$

The domain $D(\mathcal{A})$ is the subspace of $L$ for which this limit exists.

If $\mathcal{T}_{t}$ is a strongly continuous contraction semigroup, we can reconstruct $\mathcal{T}_{t}$ using its infinitesimal generator $\mathcal{A}$ (see Ethier and Kurtz (1986), Proposition 2.7 of Chapter 1). Thus the Markov process can be parameterized using $\mathcal{A}$.

The space $\mathcal{C}$ of continuous functions on a compact state space endowed with the sup norm is a usual domain for a semigroup. For instance, the generator $\mathcal{A}_{d}$ of a multivariate diffusion process is an extension of the second-order differential operator

$$
\mathcal{A}_{d} \phi(x) \equiv \frac{d}{d t} E_{x}\left[\phi\left(X_{t}\right)\right]_{\mid t=0+}=\mu \cdot \phi_{x}+\frac{1}{2} \operatorname{tr}\left[\Sigma \phi_{x x}\right]
$$

where

$$
d x_{t}=\mu\left(x_{t}\right) d t+\Lambda\left(x_{t}\right) d B_{t}
$$

$t r$ is the trace operator, and

$$
\Lambda\left(x_{t}\right) \Lambda^{\prime}\left(x_{t}\right)=\Sigma\left(x_{t}\right)
$$

For a formal proof, see for instance, Oksendal (2003). For more details, see Ait-Sahalia et al. (2004), and Hansen and Scheinkman (2002).

The domain of this second-order differential operator is restricted to the space of twice continuously differentiable functions with compact support. 


\section{Appendix B - Proofs}

\section{Appendix B1 - Proof of Proposition B.1}

Let $W^{\theta_{t}}$ define the wealth process obtained by starting at time $t$ with futures strategy $\theta$ and translating time parameters $t$ units back to time 0 , or

$$
d W_{s}^{\theta_{t}}=a_{t+s} d s+b_{t+s}^{\prime} d B_{s}
$$

where $a_{t+s}=r X_{s}^{\theta_{t}}+\pi_{t}^{\prime} \mu_{t+s}+\theta_{t+s}^{\prime} m_{t+s}-c_{t+s}$, and $b_{t+s}=\sigma_{t+s}^{\prime} \pi_{t}+v_{t+s}^{\prime} \theta_{t+s}$. Similarly, let $X^{\theta_{t}}$ be the $t$-translate of the process $X$ defined by

$$
d X_{s}^{\theta_{t}}=\alpha_{t+s} d s+\beta_{t+s}^{\prime} d B_{s}
$$

where $\alpha_{t+s}=r X_{s}^{\theta_{t}}+\theta_{t+s}^{\prime} m_{t+s}$, and $\beta_{t+s}=v_{t+s}^{\prime} \theta_{t+s}$.

Define the value function $J: \mathbb{R}^{2} \rightarrow \mathbb{R}$ as

$$
J\left(z_{t}\right)=\max _{\theta \in \Theta, c \in V} E_{t} \int_{s \geq t}^{T}\left[f\left(c_{s}, J\left(z_{s}\right)\right)+\frac{1}{2} \kappa\left(J\left(z_{s}\right)\right) J_{z_{s}}^{\prime} \Sigma J_{z_{s}}\right] d s
$$

Notice that

$$
d Z_{t}=\mu_{z_{t}} d t+\Lambda_{z_{t}} d B_{t}
$$

where $\mu_{z_{t}}=\left(a_{t}, \alpha_{t}\right)^{\prime}$ and $\Lambda_{z_{t}}=\left(b_{t}, \beta_{t}\right)^{\prime}$.

Let $\widehat{J}: \mathbb{R}^{2} \rightarrow \mathbb{R}$ stand for the value function at the optimum. Also, define $\theta_{t}^{S D U}$ as the solution of the maximization problem, such that $z=(w, x), Z_{0}^{\theta_{t}^{S D U}} \equiv$ $\left(W_{0}^{\theta_{t}^{S D U}}, X_{0}^{\theta_{t}^{S D U}}\right)=(w, x)$, with the boundary condition $\lim _{T \rightarrow \infty} \widehat{J}\left(z_{T}\right)=0 .{ }^{24}$

The HJB equation with recursive utility is given by $u\left(c_{t}\right)-\delta J+\mathcal{A}_{d} J+$ $\frac{1}{2} \kappa(J)\left(J_{z_{t}}^{\prime} \Sigma J_{z_{t}}\right)=0$, where $\mathcal{A}_{d}$ is the infinitesimal generator ${ }^{25}$, we obtain

$$
\begin{aligned}
\delta J & =\sup _{\theta \in \mathbb{R}^{k}, c \in V}\left\{u\left(c_{t}\right)+J_{w} a_{t}+J_{x} \alpha_{t}+\right. \\
& \left.+\frac{1}{2} \operatorname{tr}\left[J_{w w} b_{t} b_{t}^{\prime}+2 J_{w x} \beta_{t} b_{t}^{\prime}+J_{x x} \beta_{t} \beta_{t}^{\prime}+\kappa(J) \sum J_{z_{t}} J_{z_{t}}^{\prime}\right]\right\}
\end{aligned}
$$

Two observations are in order here. First, this program can be split into two independent decisions once the derivative of the value function is known

$$
\sup _{c \in V}\left(u\left(c_{t}\right)-J_{w} c_{t}\right) \text { and }
$$

\footnotetext{
${ }^{24}$ In special situations, we may stray from the convention of assuming zero terminal utility (see Duffie and Epstein (1992)).

${ }^{25}$ See Appendix A for further details. See Appendix C for the derivation of the HJB equation. 


$$
\begin{aligned}
& \sup _{\theta \in \mathbb{R}^{k}}\left\{J_{w}\left(r X_{s}^{\theta_{t}}+\pi_{t}^{\prime} \mu_{t+s}+\theta_{t+s}^{\prime} m_{t+s}\right)+J_{x} \alpha_{t}+\right. \\
& \left.+\frac{1}{2} \operatorname{tr}\left[J_{w w} b_{t} b_{t}^{\prime}+2 J_{w x} \beta_{t} b_{t}^{\prime}+J_{x x} \beta_{t} \beta_{t}^{\prime}+\kappa(J) \Sigma J_{z_{t}} J_{z_{t}}^{\prime}\right]\right\}
\end{aligned}
$$

Second, notice that

a. $b_{t}^{\prime} b_{t}=\left(\sigma_{t}^{\prime} \pi_{t}+v_{t}^{\prime} \theta_{t}\right)^{\prime}\left(\sigma_{t}^{\prime} \pi_{t}+v_{t}^{\prime} \theta_{t}\right)=\pi_{t}^{\prime} \sigma_{t} \sigma_{t}^{\prime} \pi_{t}+2 \pi_{t}^{\prime} \sigma_{t} v_{t}^{\prime} \theta_{t}+\theta_{t}^{\prime} v_{t} v_{t}^{\prime} \theta_{t} \Rightarrow$ $\frac{\partial b_{t}^{\prime} b_{t}}{\partial \theta_{t}}=2 v_{t} \sigma_{t}^{\prime} \pi_{t}+2 v_{t} v_{t}^{\prime} \theta_{t}$

b. $b_{t}^{\prime} \beta_{t}=\left(\sigma_{t}^{\prime} \pi+v_{t}^{\prime} \theta_{t}\right)^{\prime} v_{t}^{\prime} \theta_{t}=\pi_{t}^{\prime} \sigma_{t} v_{t}^{\prime} \theta_{t}+\theta_{t}^{\prime} v_{t} v_{t}^{\prime} \theta_{t} \Rightarrow \frac{\partial b_{t}^{\prime} \beta_{t}}{\partial \theta_{t}}=v_{t} \sigma_{t}^{\prime} \pi_{t}+2 v_{t} v_{t}^{\prime} \theta_{t}$;

c. $\beta_{t}^{\prime} \beta_{t}=\theta_{t}^{\prime} v_{t} v_{t}^{\prime} \theta_{t} \Rightarrow \frac{\partial \beta_{t}^{\prime} \beta_{t}}{\partial \theta_{t}}=2 v_{t} v_{t}^{\prime} \theta_{t}$.

It then follows from the first-order conditions that, at the optimum, $\theta_{t}^{S D U}$ solves

$$
\begin{aligned}
0 & =\left(J_{w}+J_{x}\right) m_{t}+\left(J_{w w}+\kappa(J) J_{w}^{2}\right)\left(v_{t} \sigma_{t}^{\prime} \pi_{t}+v_{t} v_{t}^{\prime} \theta_{t}\right) \\
& +\left(J_{w x}+\kappa(J) J_{x} J_{w}\right)\left(v_{t} \sigma_{t}^{\prime} \pi_{t}+2 v_{t} v_{t}^{\prime} \theta_{t}\right)+\left(J_{x x}+\kappa(J) J_{x}^{2}\right) v_{t} v_{t}^{\prime} \theta_{t}
\end{aligned}
$$

The result now follows by collecting the terms.

\section{Appendix B2 - Proof of Proposition B.2}

The proof is the same as the one presented in the last appendix except that $\pi_{t+s}=\pi, c_{t+s}=0, \forall s, t \geq 0$, and that here we define the value function $J: \mathbb{R}^{2} \rightarrow$ $\mathbb{R}$ by

$$
J\left(z_{t}\right)=\max _{\theta \in \Theta} E\left[U\left(W_{T-t}^{\theta_{t}}\right)\right]
$$

Since we are maximizing expected utility at a future date $T$, the HJB equation in this case is a little bit different; it is given by $\mathcal{A}_{d} J=0 .{ }^{26}$

In this case, we define the boundary condition as $\widehat{J}\left(z_{T}\right)=U(w)$.

\section{Appendix B3 - Proof of Proposition B.3}

Itô's lemma applied to equation 4 gives us

$$
X_{t}^{\theta}=X_{0}^{\theta}+\int_{0}^{t}\left(r X_{s}^{\theta}+\theta_{s}^{\prime} m_{s}\right) d s+\int_{0}^{t} \theta_{s}^{\prime} v_{s} d B_{s}
$$

Recalling that $X_{0}^{\theta}$ is given and differentiating $X_{t}^{\theta}$ with respect to $X_{0}^{\theta}$

$$
\frac{\partial X_{t}^{\theta}}{\partial X_{0}^{\theta}}=1+r \int_{0}^{t} \frac{\partial X_{s}^{\theta}}{\partial X_{0}^{\theta}} d s
$$

\footnotetext{
${ }^{26}$ See Appendix C or Krylov (1980) for a proof.
} 
Define now $y_{s} \equiv \frac{\partial X_{s}^{\theta}}{\partial X_{0}^{\theta}}$. Then the expression is an ordinary differential equation whose solution is given by

$$
y_{t}=y_{0} e^{r t}, y_{0}=1
$$

Hence

$$
\frac{\partial X_{T-t}^{\theta}}{\partial X_{0}^{\theta}}=e^{r(T-t)}
$$

Again by Itô's lemma, now applied to equation 8

$$
\begin{aligned}
W_{T-t}^{\theta_{t}} & =W_{0}^{\theta_{t}}+\int_{0}^{T-t} a_{t+s} d s+\int_{0}^{T-t} b_{t+s}^{\prime} d B_{s}= \\
& =W_{0}^{\theta_{t}}+\int_{0}^{T-t} \pi^{\prime} \mu_{t+s} d s+\int_{0}^{T-t}\left(\sigma_{t+s}^{\prime} \pi\right)^{\prime} d B_{s}+X_{T-t}^{\theta_{t}}-X_{0}^{\theta_{t}}= \\
& =W_{0}^{\theta_{t}}+\pi^{\prime}\left(S_{T-t}-S_{0}\right)+X_{T-t}^{\theta_{t}}-X_{0}^{\theta_{t}}
\end{aligned}
$$

The result now follows as a consequence.

\section{Appendix B4 - Proof of Corollary B.4}

First observe that

$$
J\left(z_{t}\right)=E\left[U\left(W_{T-t}^{\theta_{t}}\right)\right]
$$

By differentiating inside the expectation, we obtain ${ }^{27}$

$$
\begin{aligned}
& 0<\frac{\partial J}{\partial w}=E\left[U^{\prime}\left(W_{T-t}^{\theta_{t}}\right) \frac{\partial W_{T-t}^{\theta_{t}}}{\partial w}\right] \\
& 0<\frac{\partial J}{\partial x}=E\left[U^{\prime}\left(W_{T-t}^{\theta_{t}}\right) \frac{\partial W_{T-t}^{\theta_{t}}}{\partial x}\right]
\end{aligned}
$$

Apply now Proposition 4.2 and the result follows.

\footnotetext{
${ }^{27}$ See the Appendix in DJ, which states sufficient conditions for differentiating inside the expectation. These conditions are met here because of our assumptions on the utility function.
} 


\section{Appendix B5 - Proof of Corollary 4.2}

Again, by differentiating inside the expectation, we obtain

$$
\begin{aligned}
\frac{\partial^{2} J}{\partial w^{2}} & =E\left[U^{\prime \prime}\left(W_{T-t}^{\theta_{t}}\right)\left(\frac{\partial W_{T-t}^{\theta_{t}}}{\partial w}\right)^{2}+U^{\prime}\left(W_{T-t}^{\theta_{t}}\right) \frac{\partial^{2} W_{T-t}^{\theta_{t}}}{\partial w^{2}}\right] \\
\frac{\partial^{2} J}{\partial x^{2}} & =E\left[U^{\prime \prime}\left(W_{T-t}^{\theta_{t}}\right)\left(\frac{\partial W_{T-t}^{\theta_{t}}}{\partial x}\right)^{2}+U^{\prime}\left(W_{T-t}^{\theta_{t}}\right) \frac{\partial^{2} W_{T-t}^{\theta_{t}}}{\partial x^{2}}\right] \\
\frac{\partial^{2} J}{\partial w \partial x} & =E\left[U^{\prime \prime}\left(W_{T-t}^{\theta_{t}}\right) \frac{\partial W_{T-t}^{\theta_{t}}}{\partial w} \frac{\partial W_{T-t}^{\theta_{t}}}{\partial x}+U^{\prime}\left(W_{T-t}^{\theta_{t}}\right) \frac{\partial^{2} W_{T-t}^{\theta_{t}}}{\partial w \partial x}\right]
\end{aligned}
$$

Since all the terms that multiply $U^{\prime}\left(W_{T-t}^{\theta_{t}}\right)$ are zero, the result follows by applying Proposition 4.2.

\section{Appendix B6 - Proof of Proposition 4.6}

Observe that

$$
\begin{aligned}
J_{w} & =-\gamma E_{t}\left[U\left(W_{T-t}^{\theta_{t}^{*}}\right) \frac{\partial W_{T-t}^{\theta_{t}^{*}}}{\partial w}\right] \text { and } \\
J_{w w} & =\gamma E_{t}\left[\gamma U\left(W_{T-t}^{\theta_{t}^{*}}\right)\left(\frac{\partial W_{T-t}^{\theta_{t}^{*}}}{\partial w}\right)^{2}-U\left(W_{T-t}^{\theta_{t}^{*}}\right) \frac{\partial^{2} W_{T-t}^{\theta_{t}^{*}}}{\partial w^{2}}\right]
\end{aligned}
$$

Since $\frac{\partial W_{T-t}^{\theta_{t}^{*}}}{\partial w}=1$, the result follows immediately.

\section{Appendix B7 - Proof of Proposition 5.1}

In this case the relevant $\mathrm{HJB}^{28}$ is

$$
\mathcal{A}_{d} J+\frac{1}{2} \kappa(J)\left(J_{z_{t}}^{\prime} \Sigma J_{z_{t}}\right)=0
$$

The proof then follows exactly the same lines of the derivation of equation 3.1.

\section{Appendix B8 - Proof of Lemma 5.1}

Since $J\left(z_{t}\right)=h^{-1}\left(E_{t}\left[h\left(U\left(W_{T}^{\theta_{t}}\right)\right)\right]\right)$, it follows that $h\left(J\left(z_{t}\right)\right)=E$ $\left[h\left(U\left(W_{T-t}^{\theta_{t}}\right)\right)\right]$. Taking the derivatives on both sides ${ }^{29}$

\footnotetext{
${ }^{28}$ See Appendix $\mathrm{C}$ for a proof.

${ }^{29}$ Again, differentiability inside the expectations are satisfied because of our assumptions on h. See DJ (1990, Appendix).
} 


$$
\begin{aligned}
& h^{\prime}(J) J_{w}=E\left[h^{\prime}\left(U\left(W_{T-t}^{\theta_{t}}\right)\right) U^{\prime}\left(W_{T-t}^{\theta_{t}}\right) \frac{\partial W_{T-t}^{\theta_{t}}}{\partial w}\right] \\
& h^{\prime}(J) J_{x}=E\left[h^{\prime}\left(U\left(W_{T-t}^{\theta_{t}}\right)\right) U^{\prime}\left(W_{T-t}^{\theta_{t}}\right) \frac{\partial W_{T-t}^{\theta_{t}}}{\partial x}\right]
\end{aligned}
$$

The second derivative for $J_{w x}$ is then

$$
\begin{aligned}
h^{\prime \prime}(J) J_{w} J_{x}+h^{\prime}(J) J_{w x} & =E\left[\left(h^{\prime \prime}\left(U\left(W_{T-t}^{\theta_{t}}\right)\right)\left[U^{\prime}\left(W_{T-t}^{\theta_{t}}\right)\right]^{2}\right.\right. \\
& \left.+h^{\prime}\left(U\left(W_{T-t}^{\theta_{t}}\right)\right) U^{\prime \prime}\left(W_{T-t}^{\theta_{t}}\right)\right) \frac{\partial W_{T-t}^{\theta_{t}}}{\partial w} \frac{\partial W_{T-t}^{\theta_{t}}}{\partial x} \\
& \left.+h^{\prime}\left(U\left(W_{T-t}^{\theta_{t}}\right)\right) U^{\prime}\left(W_{T-t}^{\theta_{t}}\right) \frac{\partial^{2} W_{T-t}^{\theta_{t}}}{\partial w \partial x}\right]
\end{aligned}
$$

Applying Proposition 4.2 this becomes

$$
\begin{gathered}
0<J_{w}=\frac{E\left[h^{\prime}\left(U\left(W_{T-t}^{\theta_{t}}\right)\right) U^{\prime}\left(W_{T-t}^{\theta_{t}}\right)\right]}{h^{\prime}(J)} \\
0<J_{x}=\left(e^{r(T-t)}-1\right) \frac{E\left[h^{\prime}\left(U\left(W_{T-t}^{\theta_{t}}\right)\right) U^{\prime}\left(W_{T-t}^{\theta_{t}}\right)\right]}{h^{\prime}(J)}= \\
=\left(e^{r(T-t)}-1\right) J_{w}
\end{gathered}
$$

$0 \quad>\quad h^{\prime \prime}(J) J_{w}^{2}+h^{\prime}(J) J_{w w}=$

$$
\begin{array}{ll}
= & E\left[h^{\prime \prime}\left(U\left(W_{T-t}^{\theta_{t}}\right)\right)\left[U^{\prime}\left(W_{T-t}^{\theta_{t}}\right)\right]^{2}+h^{\prime}\left(U\left(W_{T-t}^{\theta_{t}}\right)\right) U^{\prime \prime}\left(W_{T-t}^{\theta_{t}}\right)\right] \Longrightarrow \\
\Longrightarrow & J_{w w}+\kappa(J) J_{w}^{2} \\
=\frac{E\left[h^{\prime \prime}\left(U\left(W_{T-t}^{\theta_{t}}\right)\right)\left[U^{\prime}\left(W_{T-t}^{\theta_{t}}\right)\right]^{2}+h^{\prime}\left(U\left(W_{T-t}^{\theta_{t}}\right)\right) U^{\prime \prime}\left(W_{T-t}^{\theta_{t}}\right)\right]}{h^{\prime}(J)} \\
282 \\
\text { Brazilian Review of Econometrics 26(2) Nov } 2006
\end{array}
$$




$$
\begin{aligned}
0 & >h^{\prime \prime}(J) J_{x}^{2}+h^{\prime}(J) J_{x x}=\left(e^{r(T-t)}-1\right)^{2} \times \\
& =E\left[h^{\prime \prime}\left(U\left(W_{T-t}^{\theta_{t}}\right)\right)\left[U^{\prime}\left(W_{T-t}^{\theta_{t}}\right)\right]^{2}+h^{\prime}\left(U\left(W_{T-t}^{\theta_{t}}\right)\right) U^{\prime \prime}\left(W_{T-t}^{\theta_{t}}\right)\right] \Longrightarrow \\
& \Longrightarrow \kappa(J) J_{w}^{2}+\frac{J_{x x}}{\left(e^{r(T-t)}-1\right)^{2}}=J_{w w}+\kappa(J) J_{w}^{2} \Longrightarrow \\
& \Longrightarrow J_{x x}=\left(e^{r(T-t)}-1\right)^{2} J_{w w} \\
& >h^{\prime \prime}(J) J_{w} J_{x}+h^{\prime}(J) J_{w x}=\left(e^{r(T-t)}-1\right) \times \\
& =E\left[h^{\prime \prime}\left(U\left(W_{T-t}^{\theta_{t}}\right)\right)\left[U^{\prime}\left(W_{T-t}^{\theta_{t}}\right)\right]^{2}+h^{\prime}\left(U\left(W_{T-t}^{\theta_{t}}\right)\right) U^{\prime \prime}\left(W_{T-t}^{\theta_{t}}\right)\right] \Longrightarrow \\
& \Longrightarrow \kappa(J) J_{w}^{2}+\frac{h^{\prime}(J) J_{w x}}{\left(e^{r(T-t)}-1\right)}=J_{w w}+\kappa(J) J_{w}^{2} \Longrightarrow \\
\Longrightarrow & J_{x w}=\left(e^{r(T-t)}-1\right) J_{w w}
\end{aligned}
$$




\section{Appendix C - Hamilton-Jabobi-Bellman Equation}

In this subsection we derive the relevant HJB equations for our problems.

Let us first state the Hille-Yosida Theorem

Theorem 1 Let $\mathcal{T}_{t}$ be a strongly continuous contraction semigroup on $L$ with generator $\mathcal{A}$. Then

$$
(\delta \mathcal{I}-\mathcal{A})^{-1} g=\int_{0}^{\infty} e^{-\delta t} \mathcal{T}_{s} g d s
$$

for all $g \in L$ and $\delta>0$.

Proof See Ethier and Kurtz (1986), Proposition 2.1 of Chapter 1.

\section{Standard Additive Utility}

If we apply this theorem to a standard additive utility function, say, $J\left(z_{t}\right)=$ $E_{t}\left[\int_{s \geq t} e^{-\delta(s-t)} u\left(c_{s}\right) d s\right], t \geq 0$, we then obtain the following (standard) HJB equation

Proposition C.1 Assume $u \in L$ and $\delta>0$. The HJB equation is then

$$
u\left(c_{t}\right)-\delta J+\mathcal{A}_{d} J=0
$$

Proof (One may alternatively just apply the theorem) Define the value function as

$$
J\left(z_{t}\right)=E_{t}\left[\int_{s \geq t} e^{-\delta(s-t)} u\left(c_{s}\right) d s\right], t \geq 0
$$

We then have

$$
J\left(z_{t+\varepsilon}\right)=E_{t+\varepsilon}\left[\int_{s \geq t+\varepsilon} e^{-\delta(s-t-\varepsilon)} u\left(c_{s}\right) d s\right], t+\varepsilon \geq 0
$$

Taking the conditional expectations at $t$

$$
\mathcal{T}_{\varepsilon} J\left(z_{t}\right)=E_{t}\left[\int_{s \geq t+\varepsilon} e^{-\delta(s-t-\varepsilon)} u\left(c_{s}\right) d s\right]
$$

Subtracting the first equation from the last one

$$
\mathcal{T}_{\varepsilon} J\left(z_{t}\right)-J\left(z_{t}\right)=E_{t}\left[\int_{s \geq t+\varepsilon} e^{-\delta(s-t-\varepsilon)} u\left(c_{s}\right) d s-\int_{s \geq t} e^{-\delta(s-t)} u\left(c_{s}\right) d s\right]
$$


Dividing by $\varepsilon$ and taking the limit when $\varepsilon \downarrow 0$

$$
\lim _{\varepsilon \downarrow 0} \frac{L H S}{\varepsilon}=\lim _{\varepsilon \downarrow 0} \frac{\mathcal{T}_{\varepsilon} J\left(z_{t}\right)-J\left(z_{t}\right)}{\varepsilon}=\mathcal{A}_{d} J
$$

Let us now analyze the RHS

$$
\begin{aligned}
R H S & =E_{t}\left[\int_{s \geq t+\varepsilon} e^{-\delta(s-t-\varepsilon)} u\left(c_{s}\right) d s\right]-E_{t}\left[\int_{s \geq t} e^{-\delta(s-t)} u\left(c_{s}\right) d s\right]= \\
& =E_{t}\left[\int_{s \geq t+\varepsilon} e^{-\delta(s-t)}\left(e^{\delta \varepsilon} u\left(c_{s}\right)-u\left(c_{s}\right)\right) d s-\int_{t}^{t+\varepsilon} e^{-\delta(s-t)} u\left(c_{s}\right) d s\right]
\end{aligned}
$$

Dividing by $\varepsilon$ and taking the limit when $\varepsilon \downarrow 0$

$$
\begin{aligned}
& \lim _{\varepsilon \downarrow 0} \frac{R H S}{\varepsilon} \\
= & \lim _{\varepsilon \downarrow 0} \frac{E_{t}\left[\int_{s \geq t+\varepsilon} e^{-\delta(s-t)}\left(e^{\delta \varepsilon} u\left(c_{s}\right)-u\left(c_{s}\right)\right) d s-\int_{t}^{t+\varepsilon} e^{-\delta(s-t)} u\left(c_{s}\right) d s\right]}{\varepsilon} \\
= & \lim _{\varepsilon \downarrow 0} E_{t}\left[\delta \int_{s \geq t+\varepsilon} e^{-\delta(s-t)} e^{\delta \varepsilon} u\left(c_{s}\right) d s-\left(u\left(c_{t+\varepsilon}\right)-e^{-\delta \varepsilon} u\left(c_{t+\varepsilon}\right)\right)\right]-u\left(c_{t}\right) \\
= & \delta J\left(z_{t}\right)-u\left(c_{t}\right)
\end{aligned}
$$

(Note that we have used Hospital's rule on the second line.) The result follows immediately.

\section{Standard Recursive Utility}

The same proof may be applied to the case of recursive utility,

$$
E_{t} \int_{s \geq t}\left[f\left(c_{s}, J\left(z_{s}\right)\right)+\frac{1}{2} \kappa\left(J\left(z_{s}\right)\right) J_{z_{s}}^{\prime} \Sigma J_{z_{s}}\right] d s
$$

Proposition C.2 Assume $f, k \in L$ and $\delta>0$. The HJB equation is then

$$
u\left(c_{t}\right)-\delta J+\mathcal{A}_{d} J+\frac{1}{2} k(J) J_{z_{t}}^{\prime} \Sigma J_{z_{t}}=0
$$

Proof The idea is the same as before. We just have to examine what happens to

$$
E_{t} \int_{s \geq t+\varepsilon} \frac{1}{2} \kappa(J) J_{z_{s}}^{\prime} \Sigma J_{z_{s}} d s-E_{t} \int_{s \geq t} \frac{1}{2} \kappa(J) J_{z_{s}}^{\prime} \Sigma J_{z_{s}} d s=
$$




$$
=-E_{t} \int_{t}^{t+\varepsilon} \frac{1}{2} \kappa(J) J_{z_{s}}^{\prime} \Sigma J_{z_{s}} d s
$$

Dividing by $\varepsilon$ and taking the limit when $\varepsilon \downarrow 0$

$$
-\lim _{\varepsilon \downarrow 0} \frac{E_{t} \int_{t}^{t+\varepsilon} \frac{1}{2} \kappa(J) J_{z_{s}}^{\prime} \Sigma J_{z_{s}} d s}{\varepsilon}=-\frac{1}{2} \kappa(J) J_{z_{t}}^{\prime} \Sigma J_{z_{t}}
$$

See Duffie and Epstein (1992) for another approach to the derivation. For a heuristic proof take

$$
J\left(z_{t}\right)=\varepsilon u\left(c_{t}\right)+e^{-\delta \epsilon} h^{-1}\left(\mathcal{T}_{\epsilon} h(J)\right)
$$

Then

$$
\begin{aligned}
0 & =\lim _{\varepsilon \downarrow 0}\left[u\left(c_{t}\right)+\frac{e^{-\delta \epsilon} h^{-1}\left(\mathcal{T}_{\epsilon} h(J)\right)-J}{\epsilon}\right]= \\
& =\lim _{\varepsilon \downarrow 0}\left[u\left(c_{t}\right)+\frac{-\delta e^{-\delta \epsilon} h^{-1}\left[\mathcal{T}_{\epsilon} h(J)\right]+e^{-\delta \epsilon} \frac{\mathcal{A}_{d} h(J)}{h^{\prime}(J)}}{1}\right]= \\
& =u\left(c_{t}\right)-\delta J\left(z_{t}\right)+\frac{\mathcal{A}_{d} h(J)}{h^{\prime}(J)}
\end{aligned}
$$

The proof now follows along the same lines as in Proposition 6.5.

\section{Degenerated Additive Utility}

We use the same kind of reasoning as above.

Proposition C.3 The relevant HJB equation which maximizes $J\left(z_{t}\right)=$ $E_{t}\left[U\left(W_{T}^{\theta_{t}}\right)\right]$ is given by

$$
\mathcal{A}_{d} J=0
$$

Proof This is trivial. Just note that $J\left(z_{t}\right)=E_{t}\left[U\left(W_{T}^{\theta_{t}}\right)\right]$, implying

$$
J\left(z_{t+\varepsilon}\right)=E_{t+\varepsilon}\left[U\left(W_{T}^{\theta_{t+\varepsilon}}\right)\right]
$$

Thus 


$$
\mathcal{T}_{\varepsilon} J\left(z_{t}\right)-J\left(z_{t}\right)=0
$$

And the proof follows the LHS of the second Proposition in the next section.

See Krylov (1980), Chapter 5 for another approach to the derivation.

\section{Modified Degenerated Additive Utility}

Here we can apply either of two equivalent specifications.

Proposition C.4 Consider the following value function

$$
J\left(z_{t}\right)=E_{t}\left[U\left(W_{T}^{\theta_{t}}\right)\right]+\frac{1}{2} E_{t} \int_{s \geq t}^{T} \kappa\left(J\left(z_{s}\right)\right) J_{z_{s}}^{\prime} \Sigma J_{z_{s}} d s
$$

with boundary condition $J\left(z_{T}\right)=U(w)$.

The relevant $H J B$ equation is then

$$
\mathcal{A}_{d} J+\frac{1}{2} \kappa(J) J_{z_{t}}^{\prime} \Sigma J_{z_{t}}=0
$$

Proof See the earlier proofs.

We could alternatively specify the following proposition.

Propostion C.5 Consider now the value function given by

$$
\begin{gathered}
J\left(z_{t}\right)=h^{-1}\left(E_{t}\left[h\left(U\left(W_{T}^{\theta_{t}}\right)\right)\right]\right) \\
J\left(z_{T}\right)=U(w)
\end{gathered}
$$

The relevant $H J B$ equation is then

$$
\mathcal{A}_{d} J+\frac{1}{2} \kappa(J) J_{z_{t}}^{\prime} \Sigma J_{z_{t}}=0
$$

Proof Observe that

$$
h\left(J\left(z_{t}\right)\right)=E_{t}\left[h\left(U\left(W_{T}^{\theta_{t}}\right)\right)\right]
$$

We now follow the same procedure as before.

$$
h\left(J\left(z_{t+\varepsilon}\right)\right)=E_{t+\varepsilon}\left[h\left(U\left(W_{T}^{\theta_{t+\varepsilon}}\right)\right)\right]
$$


Taking the conditional expectations at $t$ and subtracting the first equation

$$
\mathcal{T}_{\varepsilon} h\left(J\left(z_{t}\right)\right)-h\left(J\left(z_{t}\right)\right)=0
$$

Thus

$$
\lim _{\varepsilon \downarrow 0} \frac{L H S}{\varepsilon}=\lim _{\varepsilon \downarrow 0} \frac{\mathcal{T}_{\varepsilon} h\left(J\left(z_{t}\right)\right)-h\left(J\left(z_{t}\right)\right)}{\varepsilon}=\mathcal{A}_{d} h\left(J\left(z_{t}\right)\right)=0
$$

Since $\frac{\partial h(J)}{\partial z}=h^{\prime}(J) J_{z}$ and $\frac{\partial^{2} h(J)}{\partial z \partial z^{\prime}}=h^{\prime \prime}(J) J_{z} J_{z}^{\prime}+h^{\prime}(J) J_{z z}$, we then have

$$
\begin{aligned}
0 & =\frac{\mathcal{A}_{d} h(J)}{h^{\prime}(J)}=\frac{1}{h^{\prime}(J)}\left[\mu \cdot \frac{\partial h}{\partial z_{t}}+\frac{1}{2} \operatorname{tr}\left(\Sigma \frac{\partial^{2} h(J)}{\partial z_{t} \partial z_{t}^{\prime}}\right)\right]= \\
& =\mu \cdot J_{z_{t}}+\frac{1}{2} \operatorname{tr}\left(\Sigma J_{z_{t} z_{t}}\right)+\frac{h^{\prime \prime}(J)}{2 h^{\prime}(J)}\left[J_{z_{t}}^{\prime} \Sigma J_{z_{t}}\right]= \\
& =\mathcal{A}_{d} J+\frac{1}{2} \kappa(J) J_{z_{t}}^{\prime} \Sigma J_{z_{t}}
\end{aligned}
$$

It then follows that

$$
0=\frac{\mathcal{A}_{d} h(J)}{h^{\prime}(J)}=\mathcal{A}_{d} J\left(z_{t}\right)+\frac{1}{2} \kappa(J) J_{z_{t}}^{\prime} \Sigma J_{z_{t}}
$$




\section{Appendix E - Risk Aversion Increases with Power Utility}

Proposition E.1 With power utility and exponential risk adjustment.

Proof $\operatorname{cov}\left(e^{-\rho U},\left(W_{T-t}^{\theta_{t}}\right)^{-a-\gamma}\right), a=0,1$, is positive, since $\frac{d}{d W} \exp \left[-\rho \frac{W^{1-\gamma}}{1-\gamma}\right]<$ 0 , and $\frac{d}{d W} W^{-a-\gamma}<0$. Now, in order to simplify the notation, let $q \equiv e^{-\rho U}$, $p \equiv\left(W_{T-t}^{\theta_{t}}\right)^{-1-\gamma}$, and $g \equiv\left(W_{T-t}^{\theta_{t}}\right)^{-\gamma}$. We want to prove that

$$
\frac{E(p q)}{E(g q)} \geq \frac{E(p)}{E(g)}
$$

Since

$$
\operatorname{Cov}(p, q) \geq 0 \Longrightarrow E(p q) \geq E(p) E(q)
$$

The same holds for $E(\mathrm{gq})$. Now, because each expectation is positive

$$
\begin{aligned}
& E(g) E(p q) \geq E(p) E(q) E(g) \\
& E(p) E(g q) \geq E(p) E(q) E(g)
\end{aligned}
$$

Subtract one from the other and the assertion is proved. 